Review

\title{
Chandrayaan-2: India's Second Lunar Exploration Mission
}

\author{
Santosh Kosambe \\ Independent Researcher, Pune, India
}

Article history

Received: 18-09-2019

Revised: $28-10-2019$

Accepted: 29-10-2019

Email: santo2040@gmail.com

\begin{abstract}
The Moon has always been the center of attention for mankind, more than any other heavenly body in the night sky. With the early history of the solar system etched on it, the Moon challenges mankind from time immemorial to discover its secrets and admire its marvels. Understanding the Moon will provide us a pathway to unravel the early evolution of the solar system and that of planet Earth. Almost twelve years after its first lunar exploration mission, India bounced back towards Moon with Chandrayaan-2. India's most advanced engineering marvel Chandrayaan-2 is on a mission unlike any before. Leveraging nearly a decade of scientific research and engineering development, India's second lunar expedition will shed light on a completely unexplored region of the Moon, its South Polar Region. The indigenously built Chandrayaan-2 consists of an orbiter, a lander (Vikram) along with a rover (Pragyan). With the payload of completely home-grown technologies, it will help us to gain a better understanding of the origin and evolution of the Moon by conducting detailed topographical studies, comprehensive mineralogical analysis and a host of other experiments. The Chandrayaan-2 is India's first robotic exploration mission to attempt a soft landing on the South Polar Region of the Moon. With the success of this mission, India became the fifth country ever to attempt a soft landing on the lunar surface. This paper provides an insight into the Chandrayaan-2 Mission. Additionally, an overview of the mission profile, challenges involved, launch vehicle architecture, spacecraft configuration and scientific payloads are also presented.
\end{abstract}

Keywords: Chandrayaan-2, ISRO, India, Lunar Exploration, Orbiter, Lander, Rover

\section{Introduction}

The Moon is the largest and brightest celestial object present in the night sky. As the only natural satellite of the Earth, it has been a subject of study to scientists and researchers all over the world. Though there are various theories about the formation of the Moon, the most promising one is the giant impact hypothesis. Astronomers believe that the Moon was formed about 4.5 billion years ago when a large Mars-sized celestial object collided with Earth. The collision involves a huge amount of energy that throws chunks of Earth's crust into space where the gravity bound the debris together to form the Moon. The exploration of the Moon began with Luna 1, a first flyby mission launched by the Soviet Union in January 1959. Although Luna 1 did not reach the Moon's surface and flew within about 5,995 km from it. Since then various space fairing nations launched several exploration missions to study the Moon. In between 1959 to 1976 , the Soviet Union launched several robotic spacecraft missions either as an orbiter or a lander to explore the Moon. The Luna program accomplished many achievements throughout its exploration. On September 13, 1959, Luna 2 became the first spacecraft to land on the lunar surface making an impact. Whereas Luna 9 became the first spacecraft to achieve a controlled soft landing on the lunar surface and Luna 10 became the first spacecraft to orbit around the Moon. Following the Soviet Union, the United States also launched several exploration missions to the Moon. In between 1961 to 1965 , nine Ranger spacecraft's were launched straight toward the Moon to capture as many images as possible before striking on its surface. NASA achieved its first controlled landing on the lunar surface with the Surveyor Program (1966-1968). The Lunar Orbiter Program (1966-1967) helped NASA to identify 
the potential landing sites for the Apollo Missions by mapping the lunar surface. The United States became the first country ever to land the first humans on the lunar surface with the Apollo Program (1969-1972).

On October 22, 2008 India participated in the race of lunar exploration with the launch of Chandrayaan-1, the first planetary exploration mission of India. With the Indian as well as International payloads on-board, Chandrayaan-1 collected very important data during its mission of ten months. The most significant findings from Chandrayaan-1 includes, discovery of presence of hydroxyl $(\mathrm{OH})$ and water $\left(\mathrm{H}_{2} \mathrm{O}\right)$ molecules on the lunar surface and enhanced abundance towards the polar region, validating the global lunar magma ocean hypothesis, signatures of water ice present under the permanently shadowed regions near the poles, detection and mapping of reflected solar wind protons and identification of mini-magnetosphere, possible existence of water molecules in lunar environment, presence of Magnesium, Aluminum, Silicon and Calcium at several locations on the lunar surface, discovery of new rock types with unique chemical composition and radiation environment en-route and around the Moon (VSSC, 2019).

After the success of Chandrayaan-1, India launched its second mission to the Moon in the form of Chandrayaan-2. The launch of Chandrayaan-2 took place atop a GSLV MK-III M1 on July 22, 2019 at 2:43 PM Indian Standard Time (IST) from the Second Launch Pad (SLP) at the Satish Dhawan Space Centre (SDSC)
SHAR, Sriharikota. The launch of GSLV MK-III M1 is captured in Fig. 1. After sixteen minutes and fourteen seconds after the launch, the vehicle-injected Chandrayaan-2 spacecraft into a highly elliptical orbit of $169.7 \times 45,475 \mathrm{~km}$. The solar arrays mounted on the outer side of the spacecraft gets deployed immediately after the separation from the launch vehicle and ISRO Telemetry, Tracking and Command Network (ISTRAC), Bengaluru took control of the spacecraft. The Chandrayaan-2 mission will help us gain a better understanding of the origin and evolution of the Moon by conducting detail topographical studies, comprehensive mineralogical and morphological analyses and a host other experiments on the lunar surface. Also, it will enhance our capabilities by addressing some of the questions raised by the discoveries of Chandrayaan-1 and other lunar missions. The primary objective of the mission was to demonstrate the ability to soft-land a lander and operate a rover at the South Polar Region of the Moon. Whereas, the scientific goals of the mission include detailed study of topography, seismography, mineral identification and distribution, surface chemical composition, thermophysical characteristics of top soil and composition of the tenuous lunar atmosphere. Unfortunately, during its attempt on September 7, 2019 lander (Vikram) gets deviated from its intended trajectory starting at an altitude of $2.1 \mathrm{~km}$ above the lunar surface and had lost communication with the ground station..
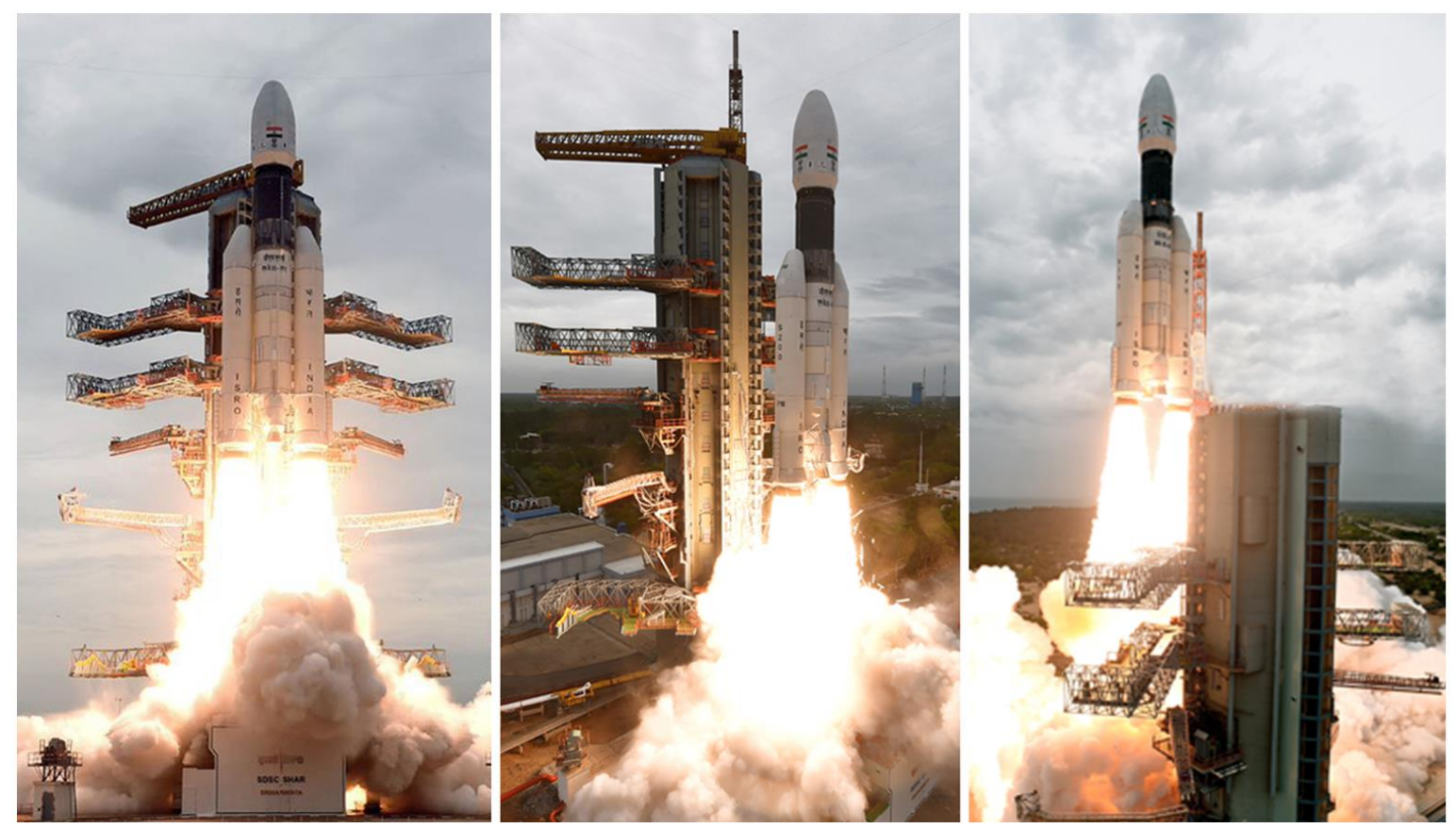

Fig. 1: Launch of GSLV MK-III M1/Chandrayaan-2 from Second Launch Pad of SDSC SHAR, Sriharikota (Credit - ISRO) 


\section{Mission Profile}

The Chandrayaan-2 Mission starts with the launch of GSLV MK-III M1. The launch vehicle successfully injected the Chandrayaan-2 spacecraft including the lunar orbiter and lander module into a highly elliptical Earth Parking Orbit (EPO) of $169.7 \times 45,475 \mathrm{~km}$. A total number of five Earth Bound Maneuvers (EBM's) and a Trans Lunar Injection (TLI) was carried out with the help of spacecraft's propulsion system to raise its orbit around the Earth until it gets placed towards the Lunar Transfer Trajectory (LTT). The performed EBM's and a TLI for Chandrayaan-2 spacecraft are shown in Table 1.

After completing a journey of 3.84 lakh $\mathrm{km}$ the spacecraft entered into Moon's sphere of influence. A final precise insertion maneuver was performed with the help of on-board thrusters to slow down the spacecraft for Lunar Capture. Again, a spacecraft performs several de-orbit maneuvers, usually called Apoapsis Adjustment Maneuvers (AAM) to change its orbit from capture orbit to a final circular orbit of $100 \times 100 \mathrm{~km}$ around the Moon.
The Lunar Orbit Insertion (LOI) and Lunar Bound Maneuvers (LBM's) executed for Chandrayaan-2 spacecraft is captured in Table 2.

The launch date and position of spacecraft upon LOI plays an important role in maximizing the lifetime of both the lander and rover. Also, the proper planning of EBM's, launch vehicle insertion parameters and lunar capture geometry with respect to Sun and Earth further extended the life of the mission. The orbital parameters such as the position of spacecraft at a specific date and time of separation were determined precisely and required corrections were made to ensure that the spacecraft was at a separation point at the predetermined time. The planned mission sequence for the Chandrayaan-2 is captured in Table 3 whereas the designed mission trajectory is presented in Fig. 2.

The separation mechanism of the spacecraft was activated at a specific location and time to separate the orbiter module from the lander module. Vikram lander separation and de-orbit operations performed are shown in Table 4.

Table 1: EBM Performed for Chandrayaan-2 Spacecraft (Credit - ISRO)

\begin{tabular}{llll}
\hline Maneuvers & Date & Time (IST) & Duration (s) \\
\hline $1^{\text {st }}$ EBM & July 24, 2019 & $14: 52$ & 48 \\
$2^{\text {nd }}$ EBM & July 26, 2019 & $01: 08$ & 883 \\
$3^{\text {rd }}$ EBM & July 29, 2019 & $15: 12$ & 989 \\
$4^{\text {th }}$ EBM & August 2, 2019 & $15: 27$ & 646 \\
$5^{\text {th }}$ EBM & August 6, 2019 & $15: 04$ & 1041 \\
TLI & August 14, 2019 & $02: 21$ & 1203 \\
\hline
\end{tabular}

Table 2: LBM Performed for Chandrayaan-2 Spacecraft (Credit - ISRO)

\begin{tabular}{lllll}
\hline Maneuvers & Date & Time (IST) & Duration (s) & Targeted Orbit (km) \\
\hline LOI/ $1^{\text {st }}$ LBM & August 20, 2019 & $09: 02$ & 1738 & $114 \times 18072$ \\
$2^{\text {nd }}$ LBM & August 21, 2019 & $12: 50$ & 1228 & $118 \times 4412$ \\
$3^{\text {rd }}$ LBM & August 28, 2019 & $09: 04$ & 1190 & $179 \times 1412$ \\
$4^{\text {th }}$ LBM & August 30, 2019 & $18: 18$ & 1155 & $124 \times 164$ \\
$5^{\text {th }}$ LBM & September 1, 2019 & $18: 21$ & 52 & $119 \times 127$ \\
\hline
\end{tabular}

Table 3: Mission sequence for chandrayaan-2 spacecraft (credit - ISRO)

\begin{tabular}{ll}
\hline Phase & Duration (After Liftoff) \\
\hline EBM's & July 22 to August 14, 2019 (23 Days) \\
TLI & August 14, 2019 \\
LTT & August 14 to August 20, 2019 (6 Days) \\
LOI & August 20, 2019 \\
LBM's & August 20 to September 1, 2019 (12 Days) \\
Lander-Orbiter Separation & September 2, 2019 \\
De-boosting & September 3 to September 4, 2019 \\
Powered Descent Starts & September 7, 2019 \\
Landing & September 7, 2019 \\
\hline
\end{tabular}

Table 4: Vikram lander separation and de-orbit operations performed for chandrayaan-2 spacecraft (Credit - ISRO)

\begin{tabular}{lllll}
\hline Maneuvers & Date & Time (IST) & Duration (s) & Targeted Orbit (km) \\
\hline Lander Separation & September 2, 2019 & $13: 15$ & - & $119 \times 127$ \\
$1^{\text {st }}$ De-orbit & September 3, 2019 & $08: 50$ & 4 & $104 \times 128$ \\
$2^{\text {nd }}$ De-orbit & September 4, 2019 & $03: 42$ & 9 & $35 \times 101$ \\
Powered Descent & September 7, 2019 & $01: 38$ & 15 & -- \\
Landing & September 7, 2019 & - & - & -- \\
\hline
\end{tabular}




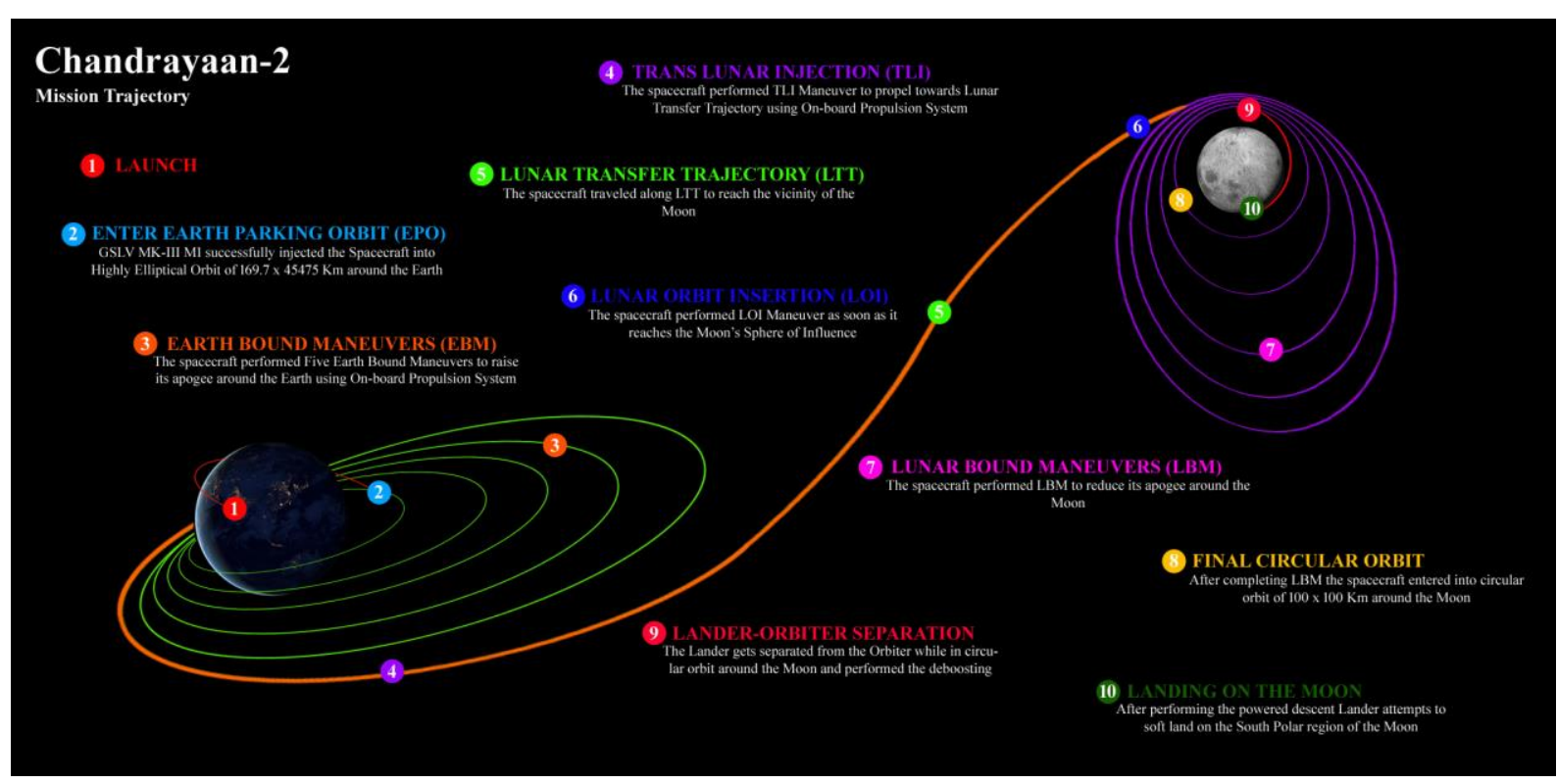

Fig. 2: Mission trajectory for chandrayaan-2 spacecraft (Credit - ISRO)

After the separation, a short burn de-boost was carried out to reduce the altitude of the lander module from 100 to $35 \mathrm{~km}$. At an altitude of $35 \mathrm{~km}$, a second longer de-boost was carried out to initiate the rough braking phase. The lander then performed a precision breaking phase to reduce the altitude further. A series of complex maneuvers comprising rough braking and fine braking was performed to eliminate the horizontal and vertical velocities associated with the lander. At an altitude of $4 \mathrm{~m}$ from the lunar surface, the thrust was cut off to free fall the lander to an impact point with a touchdown velocity greater than $5 \mathrm{~m} / \mathrm{s}$. The lander module operations from separation to touchdown at the designated landing site were carried out by a closed-loop Navigation, Guidance and Control (NGC) system installed on the lander to ensure a precise and soft landing. Onboard Guidance Algorithm takes the current position and velocity of the lander from the navigation system and generates steering profile in real-time by considering the end target states. The steering profile decides the attitude and magnitude of the thrust required for the lander. The attitude controller tracks the reference attitude while ensuring closed-loop stability. The Inertial Navigation System (INS) was prone to errors due to factors such as an error in initial states, propagation errors and inherent inaccuracies. The unbounded error growth in the INS with time was corrected with the help of updates from Absolute Navigation Sensors (ANS) which includes an INS, Star Tracker, Altimeter, Velocimeter and Image Sensor. When the lander was at a height of $6 \mathrm{~km}$ from the lunar surface, the absolute position of the lander with respect to the landing site was determined using Lander Position Detection Camera (LPDC). In addition, the horizontal and vertical velocities, absolute height with respect to Moon's surface were derived from on-board instruments and provided to the closed-loop NGC system for further refinement of trajectory. The entire descent trajectory is as shown in Fig. 3. Complete analysis of area near the landing site region prior to landing was done for finding safe and hazard-free zones. The lander (Vikram) and rover (Pragyan) was designed to carry out scientific experiments on the lunar surface for a period of one lunar day which is equal to fourteen Earth days while Orbiter will continue its mission for a duration of one year (Rekha et al., 2011).

The lander Vikram onboard Chandrayaan-2 was designed to attempt a soft landing at the south polar region of the Moon. The success of Chandrayaan-2 Mission was solely depended upon the identification and selection of specific landing site for the lander. The proper selection of landing site was essential due to some of the requirements poses by the lander because of its engineering design such as topography, temperature, environment, local and global slope, Sun illumination, radio communication, craters and boulders. The technical and scientific criteria considered for the selection of suitable landing sites includes the regions near the south pole of the Moon with slope less than $15^{\circ}$, average elevation should be greater than $2000 \mathrm{~m}$, boulders should be less than $0.5 \mathrm{~m}$, the region should not be permanently shadowed, landing ellipse area of $16 \times 10$ $\mathrm{km}$, hazard free area of $300 \times 900 \mathrm{~m}$, distance from approach side of the landing should be $12 \mathrm{~km}$ and distance exceeding the landing site of $4 \mathrm{~km}$. Based upon the above criteria some of the landing sites were envisaged using the datasets from Clementine, Lunar Reconnaissance Orbiter (LRO), Kaguya and 
Chandrayaan-1 missions. The study of data from the Lunar Orbiter Laser Altimeter (LOLA) instrument onboard LRO is of very importance to identify the potential landing sites on lunar surface for the Chandrayaan-2 mission. The possibility of water in the liquid form on the Moon is negligible due to the very high temperature of the lunar surface. Although, some of the researchers and scientists believe the possibility of the presence of water ice trapped into the Permanently Shadowed Regions (PSR) of the Moon. Hence, such PSR near or on the south pole of the Moon is of special interest to the researchers around the world.
After a detailed study of the available datasets two potential landing sites SLS54 and ALS01 which are part of the South Polar Aitken (SPA) basin were selected for the soft landing of lander Vikram. Additionally, the engineering requirements of the lander were also taken into consideration while finalizing the landing sites. The SLS54 located at $70.90^{\circ} \mathrm{S}$ and $22.78^{\circ} \mathrm{E}$ was the primary landing site for the lander. The geography associated with the primary landing site is shown in Fig. 4. Also, the alternate site as a backup for the lander ALS01 is located at $67.87^{\circ}$ $\mathrm{S}$ and $18.46^{\circ} \mathrm{W}$ (Amitabh and Srinivasan, 2018).

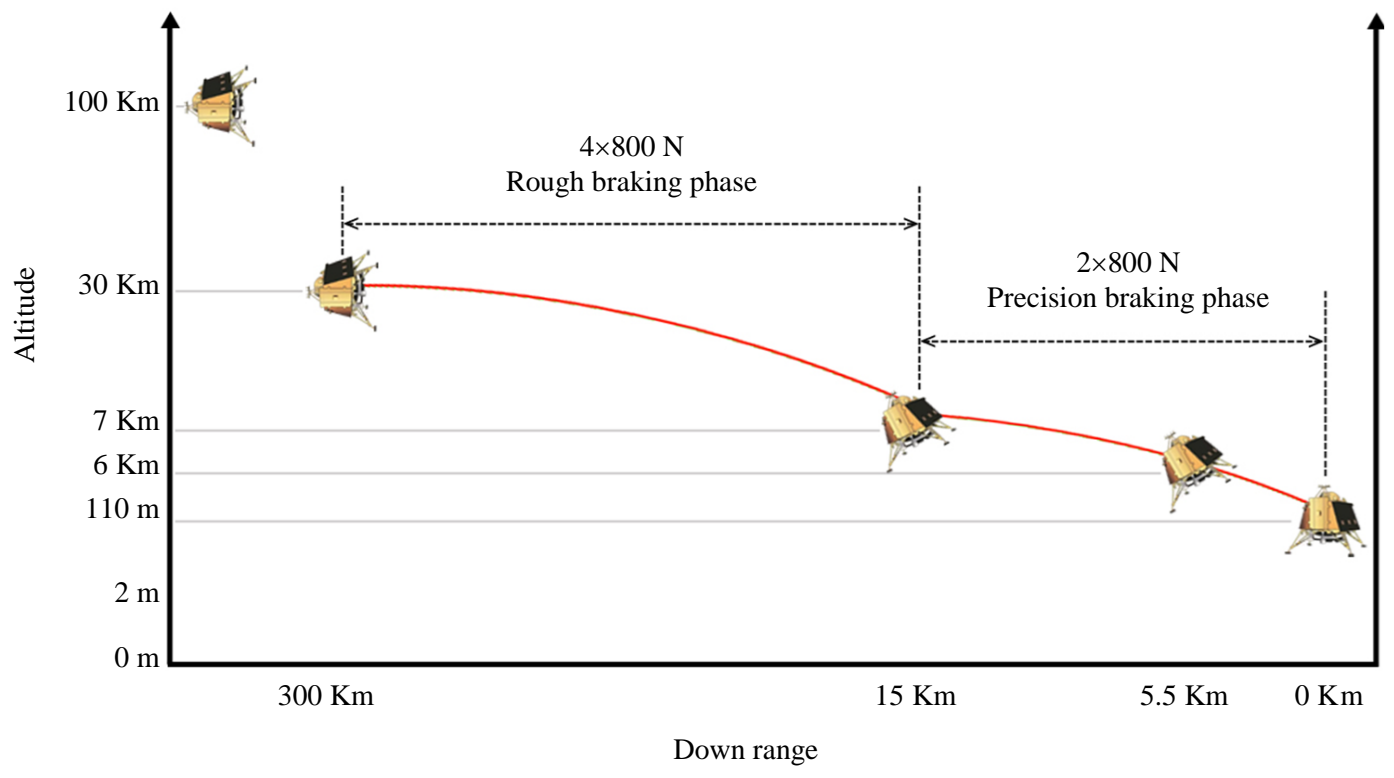

Fig. 3: Soft landing sequence for chandrayaan-2 lander module (Vikram) (Credit - ISRO)

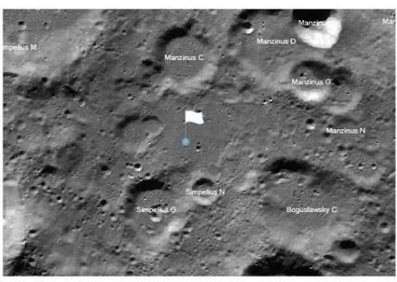

(i) LROC WAC mosaic

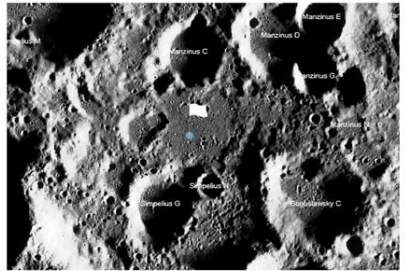

(v) LROC WAC with big shadows

LRO - Lunar Reconnaissance Orbiter WAC - Wide Angle Camera

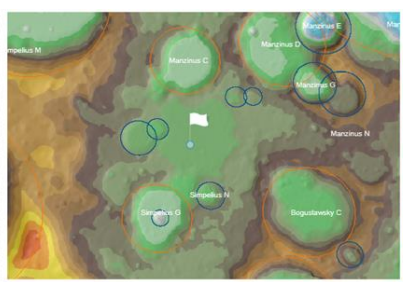

(ii) LROC WAC DTM map

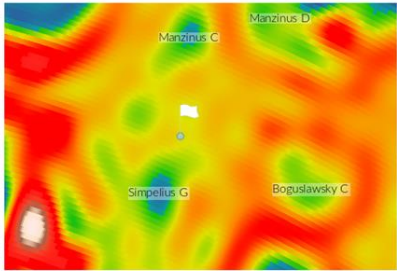

(vi) GRAIL free air gravity

LROC - Lunar Reconnaissance Orbiter Camera GRAIL - Gravity Recovery and Interior Laboratory

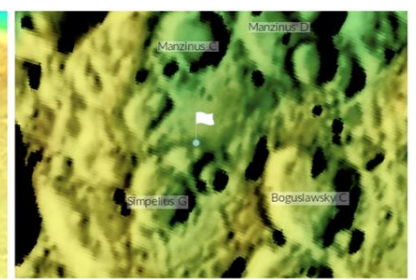

(iv) Clementine elevation map
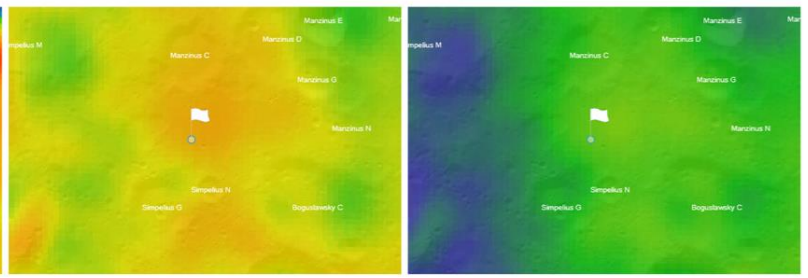

(viii) Bouguer Gravity with shading

LOLA - Lunar Orbiter Laser Altimeter DTM - Digital Terrain Model

Fig. 4: Geography of primary landing site SLS54 for chandrayaan-2 Mission (Credit - NASA) 
On September 7, 2019, Vikram lander successfully performed the powered descent consisting of rough braking and fine braking phase from an altitude of 35 $\mathrm{km}$. The powered descent was as planned and normal performance was observed up to an altitude of $2.1 \mathrm{~km}$. Subsequently, communication from the lander to the ground station was lost. Though the Vikram lander has been located by the Chandrayaan-2 Orbiter on the surface of the Moon, ISRO is unable to establish the communication with it yet.

\section{Challenges of Moon Landing}

The Lunar Exploration mission offers several challenges such as proper planning of trajectory, extreme environment and temperature variations, lumpy gravity, lunar dust and maintaining deep space communication throughout the mission. The Chandrayaan-2 Mission has overcome such numerous challenges involved in the lunar landing mission to become the fifth country to attempt a soft landing of a lander and a rover on the South Polar Region of the Moon. Some of the challenges involved in the lunar mission are discussed here.

\section{Trajectory Accuracy}

The design of a proper trajectory plays a key role in the success of any lunar missions. The primary force acting on spacecraft in space is gravity. Since gravity is such a strong force, overcoming it requires an enormous amount of energy. Also, the spacecraft is limited by the on-board fuel. Therefore, the spacecraft trajectory has to be carefully planned to avoid the expensive maneuvers required to change the orbit or velocity. As the Moon is located approximately 3.84 lakh $\mathrm{km}$ from Earth. Designing and ensuring trajectory accuracy while navigating such a large distance poses many challenges as the trajectory is influenced by the non-uniform gravity of the Earth and the Moon, gravitational pull of other astronomical bodies, solar radiation pressure and the Moon's true orbital motion (ISRO, 2019a).

\section{Deep Space Communication}

Endless communication with the Chandrayaan-2 spacecraft is essential to send commands or software updates, track spacecraft's location and velocity, to control flight path, orbit determination and estimation, to receive telemetry and scientific data, firing a rocket engine or thruster for EBM, TLI and LOI maneuvers. Due to the presence of large distance from Earth and limitation of on-board power generation, radio signals transmitted by spacecraft for communication with the Earth ground stations are very weak. The Indian Deep Space Network (IDSN) located at Byalalu near Bangalore provides deep space communication for the Chandrayaan-2 mission by picking up these weak signals from spacecraft. IDSN consists of three fully steerable 32, 18 and $11 \mathrm{~m}$ antennas to provide communication for Chandrayaan-2 spacecraft throughout its mission life (ISRO, 2019a).

\section{TLI and Lunar Capture}

The TLI is the propulsive maneuver performed by the spacecraft to propel itself on a trajectory towards the Moon. The Chandrayaan-2 spacecraft successfully performed a TLI maneuver on August 14, 2019, using the onboard propulsion system to raise its apogee successively to reach the vicinity of the Moon's orbit. As the location of Moon is changing continuously due to the presence of orbital motion, the TLI maneuver for Chandrayaan-2 spacecraft has to be timed precisely to rendezvous with the Moon. The time and duration of burn play an important role towards the success of this mission. As the Chandrayaan-2 spacecraft approaches the Moon's sphere of influence onboard thrusters are fired precisely to reduce its velocity for lunar capture. The margin of error in these calculations and maneuvers is very narrow (ISRO, 2019a).

\section{Orbiting Around the Moon}

It is observed in the past missions that when a spacecraft orbiting the Moon, passes over certain craters and impact basins, it was periodically veered off course, plummeting towards the lunar surface. The cause of such a phenomenon is the lumpy gravity present on the Moon, due to the excess distribution of mass below the lunar surface. It is also called mascons, which are large positive gravity anomalies associated with some of the giant impact basins present on the lunar surface. As the Chandrayaan-2 orbiter is planned to orbit around the Moon at an altitude of $100 \mathrm{~km}$ for a period of one year, this lumpy gravitational field will influence the orbit of the spacecraft. Since there is no atmosphere on the Moon, the precise knowledge of the thermal environment at orbital altitude is also essential for keeping on-board electronics safe and working (ISRO, 2019a).

\section{Soft Landing on the Moon}

Chandrayaan-2 is the first mission of India to attempt a soft landing at the South Polar Region of the lunar surface. The soft landing was the most challenging part of the entire mission as it requires extreme precision. The soft landing was executed by performing the rough braking and fine braking maneuvers by firing the onboard engines. Variation in the gravity at the landing site has been considered while planning lunar descent trajectory. Also, the lander was able to absorb the shock upon impact without damaging the onboard payloads and systems. The on-board NGC and propulsion system has worked together, autonomous and automatically to attempt a successful landing. Further the landing site landscape 
features should not result in a communication shadow area (ISRO, 2019a).

\section{Lunar Dust}

The lunar surface is covered with powdery grey dust due to the impact of meteorite and micrometeorite which pulverizes local rocks into fine particles. Due to the absence of atmosphere, the charged particles from the upper layers of the Sun pelted the lunar surface causing lunar soil to become electrostatically charged. This electrostatic charge made lunar soil more adhesive. As on-board engines are fired during de-boost close to the lunar surface results in a backward flow of hot gases along with dust. This dust further settles down on the lander immediately causing problems with its operations. The dust-related hazards include vision obscuration, false instrument readings, dust coating and contamination, loss of traction, clogging of mechanisms, abrasion, thermal control problems and disruption in deployment mechanism, solar panel performance and NGC sensor performance (ISRO, 2019a).

\section{Extreme Temperatures and Vacuum}

The Moon takes 27.3 days to complete one revolution around itself and also around the Earth. Due to its rotation, daytime (lunar day) on one side of the Moon lasts about 13.6 days, followed by 13.6 days of darkness (lunar night). During the lunar day, the temperature can reach $260^{\circ} \mathrm{F}$, whereas during lunar night temperature drops to $-280^{\circ} \mathrm{F}$. Also, the Diviner instrument onboard LRO recorded the lowest temperature of $-396^{\circ} \mathrm{F}$ in craters at the southern pole of the Moon. This results in extreme surface temperature variations. As Chandrayaan- 2 mission was designed to attempt a soft landing of a lander and a rover near the southern pole of the Moon, it has to withstand the extremely hot or extremely cold environment for a period of fourteen days (ISRO, 2019a).

\section{Chandrayaan-2 Launch Vehicle}

Geosynchronous Satellite Launch Vehicle Mark-III (GSLV MK-III), the largest and heaviest rocket ever built by the Indian Space Research Organization (ISRO), has launched Chandrayaan-2 to its designated orbit. The indigenously built GSLV MK-III also referred to as Launch Vehicle Mark 3 (LMV 3) is solely designed and developed by ISRO. The development program of GSLV MK-III began in early 2000 intending to launch satellites high into the geosynchronous orbit of altitude 36,000 kilometers above the equator. GSLV MK-III, ISRO's most powerful launcher to date is a $43.43 \mathrm{~m}$ tall threestage, heavy-lift launch vehicle weighing 640 tonnes. The launcher is capable of carrying a $4,000 \mathrm{~kg}$ of a satellite into Geosynchronous Transfer Orbit (GTO) and about $10,000 \mathrm{~kg}$ of a satellite into Low Earth Orbit (LEO). The specifications of GSLV MK-III M1 is exposed in Fig. 5. The three-stage launcher consists of two solid strap-ons (S200) and a core liquid booster (L110) along with a cryogenic upper stage (C25). Also, the launcher is equipped with Ogive Payload Fairing (OPLF) with a diameter of $5 \mathrm{~m}$ to accommodate heavier payloads. The first stage comprises two strap-on boosters or Large Solid Boosters (LSB) made up of three motor segments and a flex nozzle control system developed at Vikram Sarabhai Space Centre (VSSC), Thiruvananthapuram. Both the S200 boosters with a height of $25 \mathrm{~m}$ and a diameter of $3.2 \mathrm{~m}$ are located on either side of the core liquid booster. Each booster is capable of carrying 205 tons of solid propellant and is responsible for the launchers lift-off. Both the boosters can function for $140 \mathrm{sec}$ to impart the required amount of thrust for lift-off. The L110 core liquid booster with a height of $21 \mathrm{~m}$ and a diameter of $4 \mathrm{~m}$ acts as a second stage for the launcher. The core stage is capable of carrying 110 tonnes of bipropellant i.e. combination of UH25 (75\% of UDMH and $25 \%$ of Hydrazine) and Dinitrogen Tetroxide (N2O4) and is powered by twin Vikas Engines designed and developed at Liquid Propulsion Systems Centre (LPSC), Thiruvananthapuram.

Both the engines are fired after 114 seconds of lift-off and can produce 160 tons of thrust together to impart additional power to the launcher for about 322 seconds after lift-off. The third and final stage of the launcher consists of the cryogenic stage with a capacity of 28 tonnes of cryogenic propellant i.e., a combination of Liquid Oxygen (LOX) and Liquid Hydrogen (LH2) stored on-board at 77 and $20 \mathrm{~K}$ respectively. The cryogenic stage is powered by ISRO's largest cryogenic engine CE-20, designed and developed at the LPSC. The CE-20 engine works on a Gas Generator (GG) cycle and can produce 20 tonnes of nominal thrust. The $13.5 \mathrm{~m}$ tall and $4 \mathrm{~m}$ in diameter stage is integrated on top of the L110 core liquid stage. The C25 is ignited $2 \mathrm{sec}$ after the separation of the second stage and can function for a duration of 800 seconds. The first successful experimental flight of GSLV MK-III was done on December 18, 2014, in the form of LVM-X or Crew Module Atmospheric Re-entry Experiment (CARE) Mission. Several modifications in the aerodynamics of the vehicle have been incorporated after the test to improve the performance of the vehicle further. The GSLV MK-III D1/GSAT-19 Mission launched on June 5, 2017 and GSLV MK-III D2/GSAT-29 Mission marked the first and second successful developmental flight of GSLV MK-III respectively. Nearly after the 20 years of development, 11 test flights and over 200 different components tests, the GSLV MK-III is now fully operational. Further developments and modifications in the vehicle are ongoing for the Gaganyaan Mission which is ISRO's first crewed mission to be launched in December 2021 (ISRO, 2019b). The flight events associated with GSLV MK-III M1/Chandrayaan-2 Mission are captured in Table 5. 


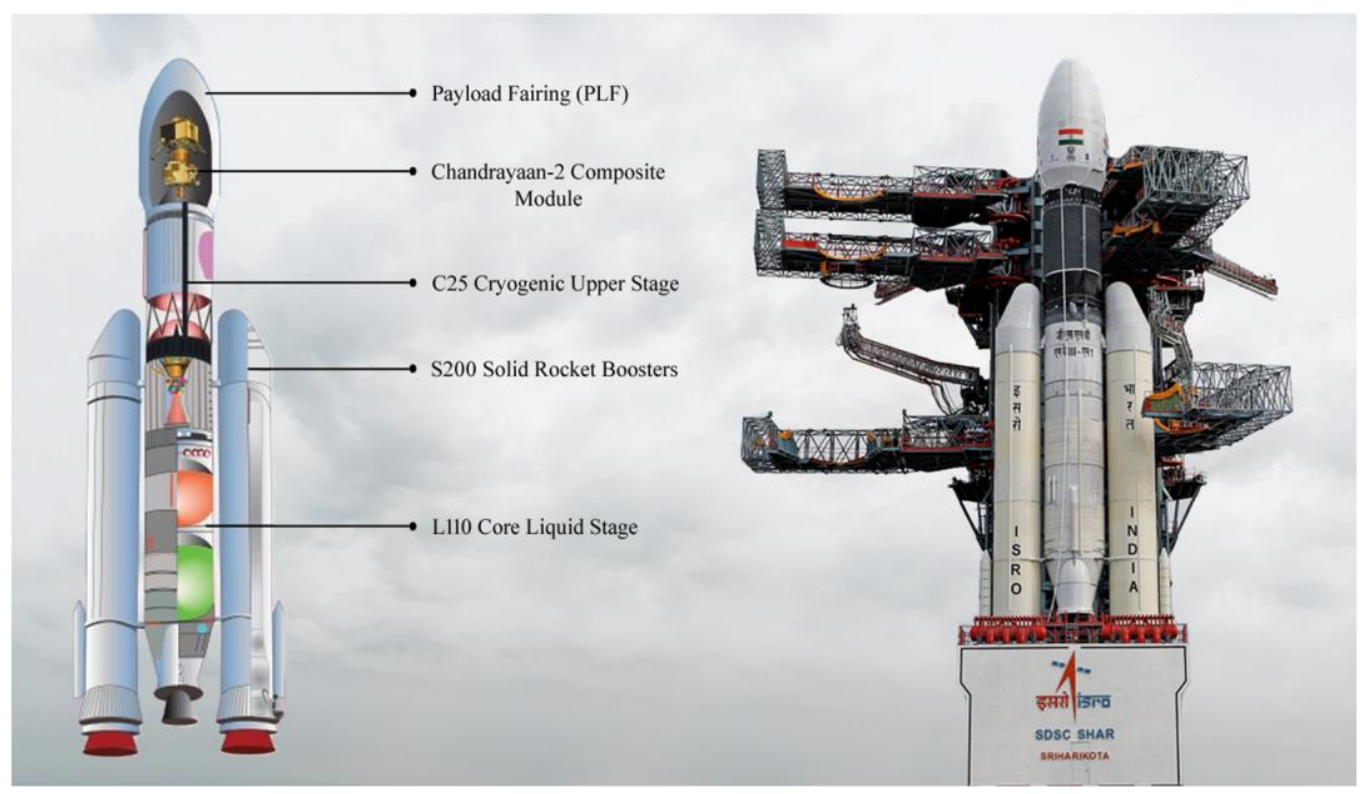

Fig. 5: Specifications of Chandrayaan-2 Launch Vehicle GSLV MK-III M1 (Credit - ISRO)

Table 5: Flight sequence for chandrayaan-2 spacecraft (credit - ISRO)

\begin{tabular}{llll}
\hline Flight events & Time $(\mathrm{s})$ & Altitude $(\mathrm{km})$ & Initial velocity $(\mathrm{m} / \mathrm{s})$ \\
\hline S200 Solid Boosters Ignition & 0.1 & 0.024 & 451.91 \\
L110 Core Liquid Stage Ignition & 112.7 & 43.939 & 1744.54 \\
S200 Solid Boosters Separation & 132.7 & 61.995 & 1956.40 \\
Payload Fairing Separation & 205.1 & 114.511 & 2620.05 \\
L110 Core Liquid Stage Shut Off & 306.4 & 169.079 & 4574.12 \\
L110 Core Liquid Stage Separation & 307.7 & 170.783 & 4610.15 \\
C25 Cryogenic Upper Stage Ignition & 313.6 & 172.072 & 4607.55 \\
C25 Cryogenic Upper Stage Shut Off & 978.7 & 176.415 & 10297.19 \\
Chandrayaan-2 Spacecraft Separation & 993.8 & 181.656 & 10305.78 \\
\hline
\end{tabular}

\section{Chandrayaan-2 Spacecraft Configuration}

Chandrayaan-2, India's second lunar mission with a mass of 3.8 tonnes and dimensions of $3.1 \times 3.1 \times 5.8 \mathrm{~m}$ comprises three modules including an Orbiter, Lander (Vikram) and Rover (Pragyan), all equipped with scientific instruments to study the only natural satellite of the Earth. As shown in Fig. 6 the Orbiter and Lander modules were interfaced mechanically and stacked together as an integrated module, whereas Rover was housed inside the Lander (ISRO, 2019b).

\section{Chandrayaan-2 Orbiter}

The Orbiter module weighing $2,397 \mathrm{~kg}$ is a $3.2 \times 5.8 \times 2.1 \mathrm{~m}$ bus structure made up of a composite cylinder, shear webs and deck panels. The orbiter is built around a cuboidal structure and houses the propulsion module including a liquid propulsion engine and onboard fuel for orbit raising maneuvers of the spacecraft. The lander separation mechanism of the launch vehicle was attached at one end of the spacecraft whereas the lander was attached at the other end. The structure of the Orbiter spacecraft was developed by Hindustan Aeronautics Limited (HAL) and delivered to the ISRO Satellite Centre (ISAC), Bangalore on June 22, 2015. The essential spacecraft subsystems and payloads were mounted onto the structure further by ISAC. The indigenously built Chandrayaan-2 Orbiter is captured in Fig. 7. The Orbiter includes several subsystems and avionics of the spacecraft. The solar array mounted on top of the spacecraft is capable of providing $1000 \mathrm{~W}$ of electric power to the spacecraft during different operational phases of the mission around the Earth and the Moon. The array consisting of two solar panels which were stowed in the launch configuration and were deployed as soon as after the separation of the spacecraft. Also, the excess amount of power will be stored inside the Lithium-Ion batteries and can be utilized during the absence of sunlight, eclipse and peak power requirements of the spacecraft. The Orbiter is designed as a three-axis stabilized spacecraft along with reaction wheels which provides a stable platform for imaging purposes. Cold Gas thruster is also mounted on a sidewall of the structure for momentum dumping and 
attitude corrections. A bipropellant liquid engine designed and developed by VSSC was used to raise the orbit of the spacecraft from EPO to $100 \times 100 \mathrm{~km}$ lunar orbit. The avionics installed on spacecraft such as star sensors, sun sensors, accelerometers and Gyros provide the attitude and orbit control data along with the body rates for controlling the spacecraft. The telemetry system provides information related to the health of the spacecraft while the tele-command system executes the command execution and distribution tasks. Several scientific payloads on the Orbiter are interfaced with the baseband data handling system for formatting and recording the data in the solid-state recorder which will be played later. The Radio-Frequency system which includes S-band TTC transponder and X-band transmitter for transmitting the spacecraft data to IDSN station situated at Byalalu. The X-band dual gimbal antenna mounted on the spacecraft and pointed towards the ground station helps in transmitting the data. The Orbiter also acts as a communication relay between the lander, rover and mission control station on Earth (Annadurai, 2017).
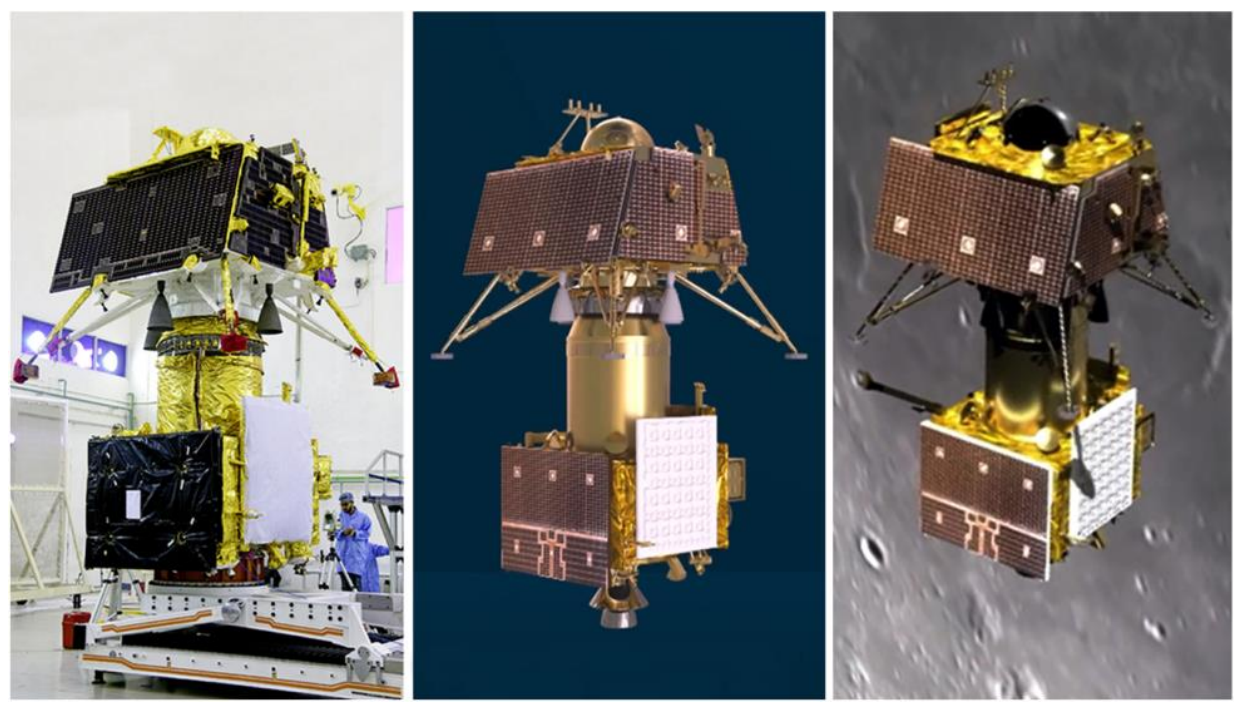

Fig. 6: Configuration of chandrayaan-2 spacecraft (Credit - ISRO)
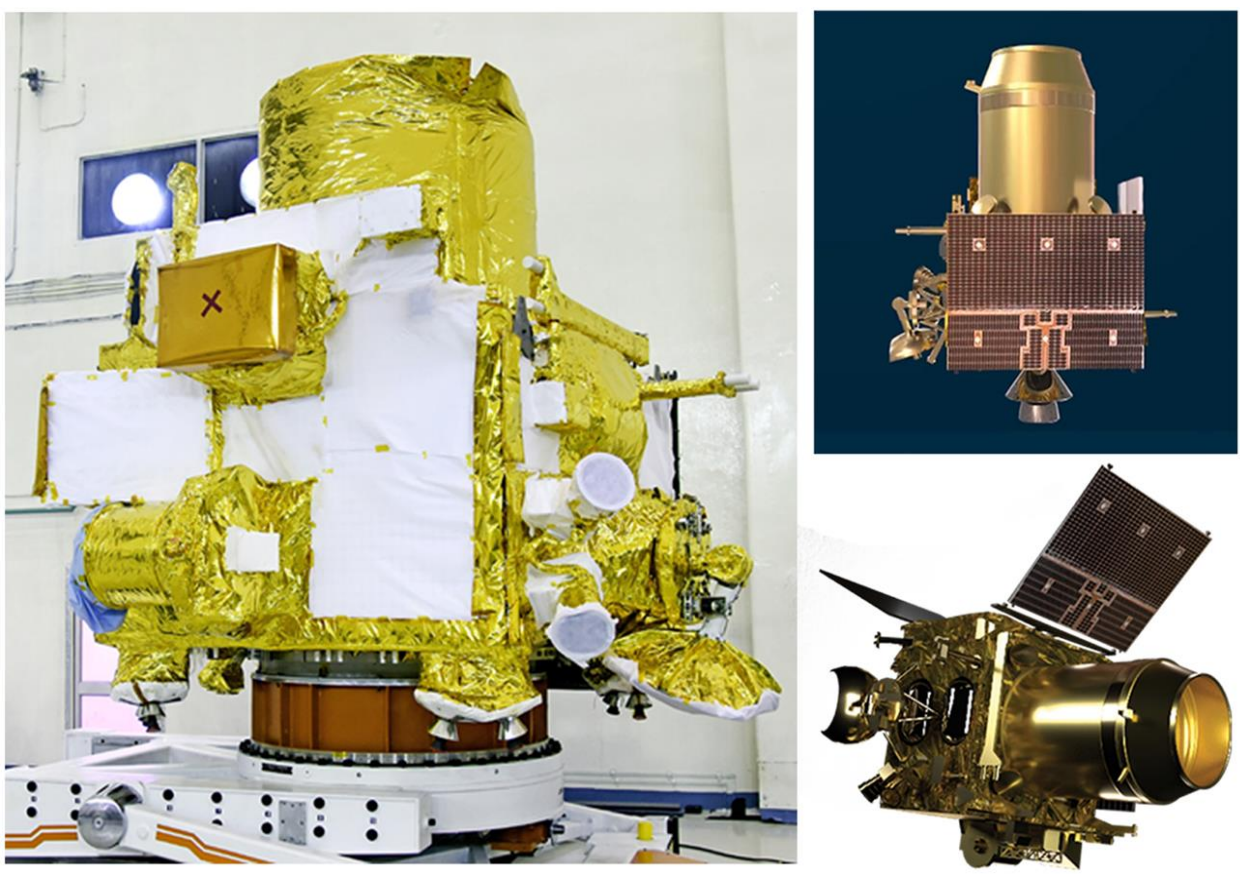

Fig. 7: Configuration of chandrayaan-2 orbiter module (Credit - ISRO) 


\section{Chandrayaan-2 Lander}

The second module of the spacecraft consists of a lander called 'Vikram' named after Dr. Vikram A Sarabhai, the founder of ISRO and the father of the Indian Space Programme. Initially, the Russian Space Agency (Roscosmos) was going to provide the lander for the mission. However, the Roscosmos withdrew from the mission after the similarly designed lander for another mission deployed problem in 2011. After that incident, ISRO took responsibility to design, develop and built a lander on its own. The initial design and preliminary configuration study of the lander were done at Space Applications Centre (SAC), Ahmedabad and later it was developed at U R Rao Satellite Centre (URSC), Bangalore. The lander module with a dimension of $2.54 \times 2 \times 1.2 \mathrm{~m}$ and weighing $1,471 \mathrm{~kg}$ along with the rover was structured as a truncated pyramid around a cylinder which houses the propellant module along with five liquid engines and propellant for performing landing operation on the surface of Moon. The Lander module developed for the mission is shown in Fig. 8. Also, the lander includes an interface for the orbiter separation mechanism which was attached at one end of the lander while the rover was housed inside of it. The solar panels mounted on top of the lander provides $650 \mathrm{~W}$ of power to the several subsystems and avionics of the lander during all phases of the mission. In addition, Lithium Ion battery stacked inside the lander provides power to the lander during the absence of sunlight, eclipse and descent operation. The four number of lander leg mechanism provides stability to the lander upon landing on different terrains of the Moon. The lander leg mechanism ensures that the energy at touchdown was absorbed and all the lander systems were integral and stable for the further conduct of payload deployments and scientific experiments on Moon. Each leg consists of a telescopic leg assembly with crushable damper material in the leg and footpad. Extensive analysis and tests were done for the lander leg mechanism to ensure stability under extreme terrain conditions and terminal velocity. The avionics and subsystems were mounted on the internal walls of the structure to protect them from the extremely cold environment. The control electronics provide an interface to all the sensors and actuator drives of the lander. The sensors were configured for providing inertial navigation to the lander starting from Orbiter separation to the end of the rough braking phase, whereas the absolute sensors were programmed to determine the accurate position and velocity with respect to the landing site to further guide the lander beyond the rough braking phase towards the identified site. The Navigation, Guidance and Control operation of the lander was totally autonomous from Orbiter separation onwards and must ensure a precise, safe and soft landing on the lunar surface. The lander was capable of performing a comprehensive check of all subsystems before attempting a soft landing on the lunar surface. The five main liquid engines, each capable of producing $800 \mathrm{~N}$ of thrust were mounted on sidewalls of the lander to provide the braking thrust while performing the decelerating operation. The attitude of the lander was controlled with the help of eight number of thrusters each capable of producing $50 \mathrm{~N}$ of thrust. All the main liquid engines, thrusters and subsystems had undergone several tests before coming together. The lander was able to communicate directly with the IDSN, the rover and the orbiter. The Telemetry, Tracking and Command (TTC) operation between the lander and IDSN was performed in S-band and the payload data was transmitted with the help of high torque dual gimbal antenna. The Telemetry and Tele-command data handling system was provided with inbuilt storage. The Rover was stowed inside of lander during launch and upon landing the ramps will be deployed and rover will start its journey on the lunar surface. The payloads mounted on the lander will be deployed after landing. The lander was designed to operate for one lunar day, which is equivalent to about 14 Earth days (Annadurai, 2017).

\section{Chandrayaan-2 Rover}

The third module of the spacecraft was a six-wheeled robotic vehicle named 'Pragyan', which means 'Wisdom' in Sanskrit. Upon landing, a six wheeled solar powered rover will detach itself and slowly crawl on the lunar surface making observations and collecting data. The rover weighing $27 \mathrm{~kg}$ and having a dimension of $0.9 \times 0.75 \times 0.85 \mathrm{~m}$ was designed with the objective of performing mobility on the low gravity and vacuum of the lunar surface and in addition to conduct scientific experiments for understanding the lunar resources. The design of the rover was based on the well proven space rover 'Sojourner' that was deployed by NASA for the exploration of the Red Planet in the form of Mars Pathfinder Mission in July 1997. All the subsystems and avionics were mounted on the chassis of the rover. The solely designed and built rover for the mission is shown in Fig. 9. The Indian Institute of Technology (IIT), Kanpur has designed, developed and validated two software algorithms which include Kinematic Control Algorithm for the rover motion on uneven terrain and algorithms for computer vision-based autonomous navigation system for mobile robots for the lunar rover mission. Two navigation cameras mounted in front of the rover utilizes stereoscopic camera-based $3 \mathrm{D}$ vision to plot the $3 \mathrm{D}$ view of the surrounding terrain to help ground control station in path planning by generating a digital elevation model of the terrain. The single solar panel mounted on one side of the rover was capable of producing and providing $50 \mathrm{~W}$ of power to the propulsion system, scientific instruments and communication instruments of the rover throughout its mission lifetime. 

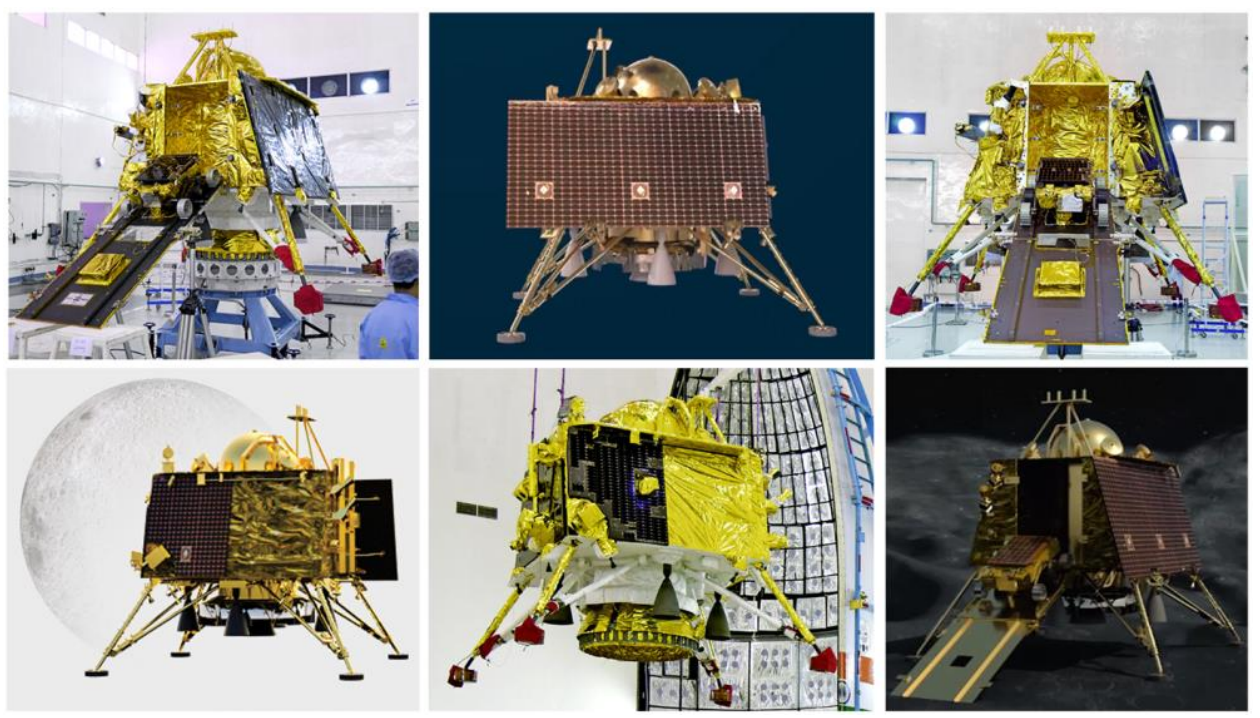

Fig. 8: Configuration of chandrayaan-2 lander module (Vikram) (Credit - ISRO)
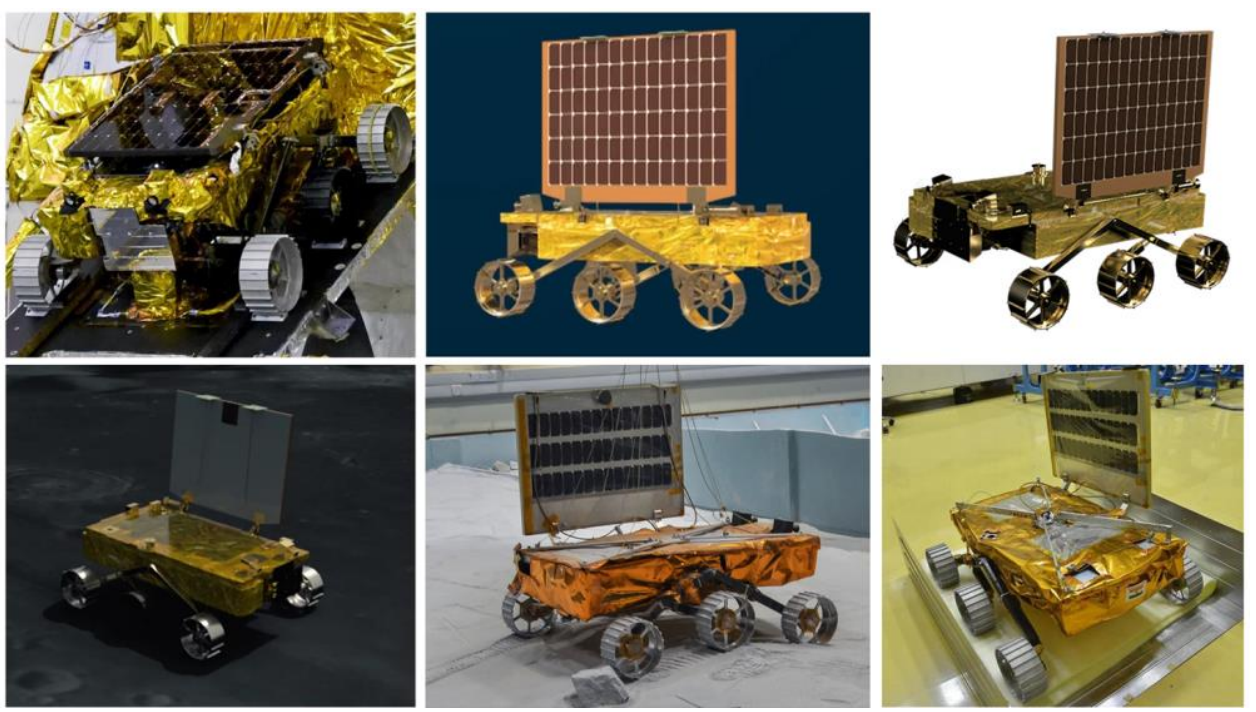

Fig. 9: Configuration of chandrayaan-2 Rover (Pragyan) (Credit - ISRO)

The rover was able to travel on the lunar surface within a $500 \mathrm{~m}$ radius of the landing site at a speed of $1 \mathrm{~cm}$ per second. The six-wheel drive, each driven by independent brushless DC electric motors was equipped with the rocker-bogie suspension mechanism having ten degrees of freedom (DOF) to ensure rugged mobility over obstacles and slopes along the identified path for exploration of the region. The front and rear wheels of the rover have steering motors and steering was accomplished by the differential speed of the wheels or skid steering. The rover was able to communicate to the IDSN via the lander. The rover was designed to operate for one lunar day i.e. nearly 14 Earth days but it can sustain for longer than planned due to the implementation of the solar-powered wake-up cycle to its power system (Annadurai, 2017).

\section{Chandrayaan-2 Scientific Payloads}

Chandrayaan-2 Mission includes several scientific instruments to expand the lunar scientific knowledge through a detailed study of topography, seismology, mineral identification and distribution, surface chemical composition, thermo-physical characteristics of top soil and composition of the tenuous atmosphere, leading to a new understanding of the origin and evolution of the Moon. The Orbiter payloads will conduct remotesensing observations from a $100 \mathrm{~km}$ orbit while the Lander and Rover payloads will perform in-situ measurements near the landing site. The scientific payloads on-board the Chandrayaan-2 spacecraft are presented in Fig. 10. 


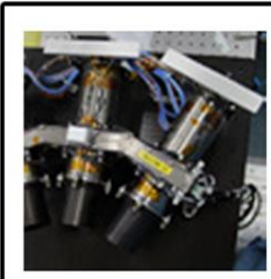

(a) TMC 2

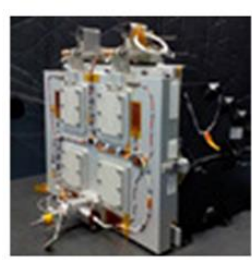

(b) CLASS

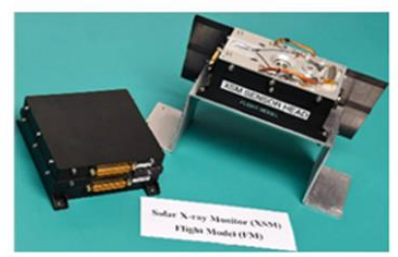

(c) XSM

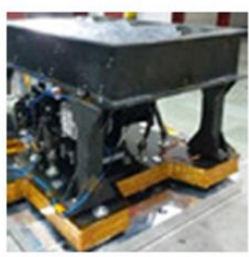

(d) OHRC

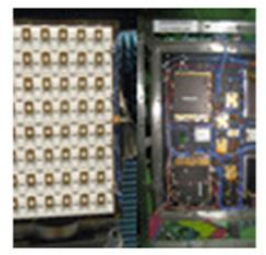

(e) IIRS

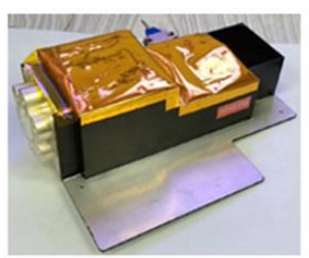

(f) APXS

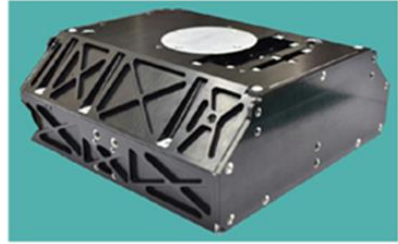

(g) ILSA

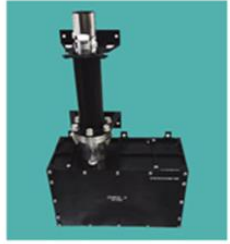

(h) CHASE 2

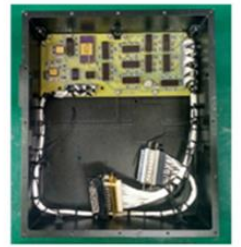

(i) DFRS

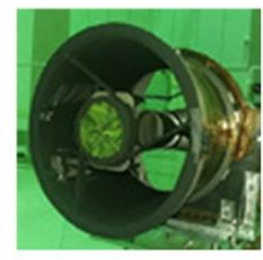

(j) OHRC

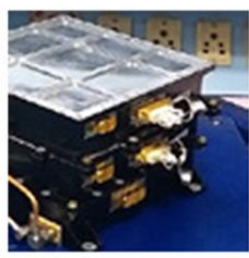

(k) DFSAR

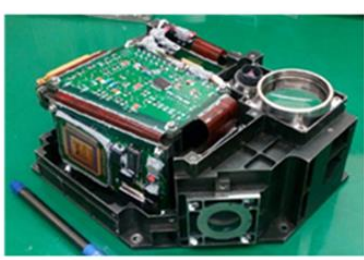

(l) LIBS

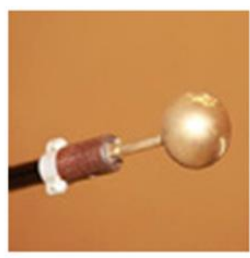

(m) RAMBHA

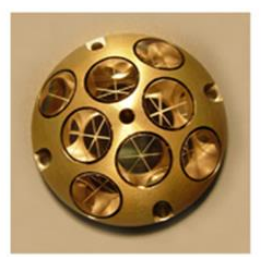

(n) LRA

Fig. 10: Scientific payloads on-board chandrayaan-2 spacecraft (Credit - ISRO)

\section{Orbiter Payloads}

The Chandrayaan-2 Orbiter consists of eight scientific payloads to study the Moon. The instruments mounted on the Orbiter are as follow:

\section{Terrain Mapping Camera 2 (TMC 2)}

TMC 2 is a miniature version of the TMC used onboard the Chandrayaan-1 Mission. The primary objective of the camera is to map the lunar surface in the panchromatic spectral band (0.5-0.8 microns) with a high spatial resolution of $5 \mathrm{~m}$ and a swath of $20 \mathrm{~km}$ from 100 $\mathrm{km}$ lunar polar orbit. The camera is designed, developed and provided by SAC, Ahmedabad. The data collected by TMC 2 will help us to find clues about the Moon's evolution and help us to plot a detailed three dimensional map of the lunar surface which is essential for studying lunar mineralogy and geology (ISRO, 2019c).

\section{Chandrayaan-2 Large Area Soft X-ray Spectrometer (CLASS)}

CLASS is a continuation of the successful C1XS XRF experiment on Chandrayaan-1. CLASS will measure the presence of major rock-forming elements such as Magnesium, Aluminum, Silicon, Calcium, Titanium, Iron and Sodium on the lunar surface using the technique of X-ray Fluorescence (XRF) spectra during solar flare events at a resolution of few tens of kilometers. The XRF will detect the presence of these elements by measuring the characteristic X-rays they emit when excited by the Sun's rays. It is designed to provide a lunar mapping of the presence of such elements with a nominal spatial resolution of $25 \mathrm{~km}$ (FWHM) from a $100 \mathrm{~km}$ lunar polar orbit. The objectives of the instrument are to make global studies on the diversity and distribution of lunar lithologies, bulk composition of the crust, abundance patterns in the major crustal provinces and mare basalt diversity. Together with mineralogical data this would provide a comprehensive picture of lunar surface chemistry. The instrument is designed, developed and provided by ISAC, Bangalore (Narendranath et al., 2014).

\section{Solar X-ray Monitor (XSM)}

XSM instrument consists of two scientific packages namely, XSM sensor package and XSM electronics package. XSM will observe the X-rays emitted by the Sun and its corona, measure the intensity of solar radiation in these rays and supports CLASS. The primary objective of XSM is to accurately calculate the solar X-ray spectrum in the energy range of $1-15 \mathrm{keV}$ with energy resolution $\sim 200 \mathrm{eV}$ at $5.9 \mathrm{keV}$. This will be achieved by using state of the art Silicon Drift Detector (SDD), which has a unique capability of maintaining high energy resolution at very high incident count rate expected from Solar X-rays. XSM will provide high- 
energy resolution and high-cadence measurements (Full spectrum every second) of solar X-ray spectra as input for analysis of data from CLASS. The XSM is designed, developed and provided by Physical Research Laboratory (PRL), Ahmedabad (Vadawale et al., 2014).

\section{Orbiter High Resolution Camera (OHRC)}

OHRC will provide high-resolution images of the Moon to ensure the presence of any craters or boulders prior to the separation of the lander. The instrument will capture the images from two different angles to serve dual purposes. Firstly, it will be utilized to plot the Digital Elevation Models (DEM) of the landing site before the separation of the lander from Orbiter. Secondly, it will be used for carrying out scientific research after the lander separation. The OHRC operates in the visible panchromatic band $(450-800 \mathrm{~nm})$. The images will be captured over the course of two orbits covering an area of $12 \times 3 \mathrm{~km}$ with a spatial resolution of $25 \mathrm{~cm}$. (ISRO, 2019c).

\section{Imaging IR Spectrometer (IIRS)}

IIRS is designed to achieve two prime objectives. The first objective of IIRS includes global mineralogical and volatile mapping of the Moon for the first time in the spectral range of $\sim 0.8$ to $5.0 \mu \mathrm{m}$ and at the high resolution of $\sim 20 \mathrm{~nm}$. The second objective is to perform complete characterization of water $\left(\mathrm{H}_{2} \mathrm{O}\right)$ and hydroxyl $(\mathrm{OH})$ feature near $3.0 \mu \mathrm{m}$ for the first time at high spatial $(\sim 80 \mathrm{~m})$ and spectral $(\sim 20 \mathrm{~nm})$ resolutions in South Polar Region. The instrument will also help to measure the solar radiation reflected off the Moon's surface in 256 contiguous spectral bands from its $100 \mathrm{~km}$ lunar polar orbit. It will also study mare volcanism, variations in basaltic compositions, mantle heterogeneity at the basin and local scale. According to ISRO, the IIRS has begun its spectroscopic study of the lunar surface and successfully acquired the first illuminated image of the lunar surface. The IIRS is designed, developed and provided by SAC, Ahmedabad (ISRO, 2019c).

\section{Dual Frequency Synthetic Aperture Radar (DFSAR)}

DFSAR will utilize the L-band and S-band to provide enhanced capabilities compared to the Chandrayaan-1 missions S-band miniSAR instrument. The DFSAR will utilize L-band to provide deeper penetration of lunar regolith up to $5 \mathrm{~m}$ which is twice that of S-band. It will also conduct circular and full polarimetry of lunar surface along with standalone ( $\mathrm{L}$ or S-band) and simultaneous ( $\mathrm{L}$ and $\mathrm{S}$-band) modes of imaging with a resolution ranging from 2 to $75 \mathrm{~m}$ and with incident angles ranging from 90 to 350 for understanding scattering properties of permanently shadowed regions especially in the south polar regions. The DFSAR is designed to estimate the regolith thickness and its distribution on the lunar surface, quantitative estimation of the presence of water ice in the SPR and high-resolution mapping of the lunar surface in SPR. The DFSAR will also help in resolving some of the ambiguities in interpreting high values of Circular Polarization Ratio (CPR) observed in miniSAR data. The added information from full-polarimetric data will allow greater confidence in the results derived particularly in detecting the presence and estimating the quantity of water ice in the polar region. The DFSAR is designed, developed and provided by SAC, Ahmedabad (Putrevu et al., 2016).

\section{Chandrayaan-2 Atmospheric Compositional Explorer 2 (CHACE 2)}

CHASE 2 mass spectrometer aboard the Chandrayaan-2 Orbiter will continue the CHACE experiment carried out by Chandrayaan-1. It will carry out a detailed study of the lunar exosphere from its 100 $\mathrm{km}$ lunar polar orbit. It includes a Quadrupole Mass Spectrometer (QMA) which is capable of scanning the lunar neutral exosphere in the mass range of 1 to 300 amu with a mass resolution of $\sim 0.5 \mathrm{amu}$. It will also cover the polar regions of the Moon including the permanently shadowed regions (PSR), which are believed to be pristine enough to retain the history of the inner solar system. By taking advantage of the axial rotation of the Moon and the polar orbit of Chandrayaan-2, the instrument will study the global distribution of the lunar exosphere. It will also study the day-night variation of the lunar neutral exosphere as well as the variation during the passage through the geomagnetic tail. The primary objective of CHACE 2 is to carry out an in-situ study of the composition and distribution of the lunar neutral exosphere and its variability. CHACE 2 is designed, developed and provided by Space Physics Laboratory (SPL), Thiruvananthapuram (Bhardwaj et al., 2016).

\section{Dual Frequency Radio Science (DFRS) Experiment}

DFRS is designed to study the temporal evolution of electron density in the lunar ionosphere. Two coherent signals of X-band with frequency $8496 \mathrm{MHz}$ and S-band with frequency $2240 \mathrm{MHz}$ is transmitted simultaneously from satellite and is received at ground based IDSN receivers (ISRO, 2019c).

\section{Lander Payloads}

The Chandrayaan-2 Lander consists of three scientific payloads to study the Moon. The instruments mounted on the Lander are as follow:

\section{Radio Anatomy of Moon Bound Hypersensitive Ionosphere and Atmosphere (RAMBHA)}

The lunar ionosphere is a highly dynamic plasma environment. A unique payload package, such as 
RAMBHA, has proven to be an effective diagnostic tool to provide a comprehensive exploration of the lunar plasma environment. It consists of a Langmuir Probe (LP) to measure the density of the lunar near-surface plasma and how it changes over time. The primary objective of RAMBHA is to measure factors such as ambient electron density and temperature near the lunar surface and temporal evolution of lunar plasma density for the first time near the surface under varying solar conditions. It is designed and developed at SPL, Thiruvananthapuram (Manju, 2016).

\section{Chandra's Surface Thermo-physical Experiment (ChaSTE)}

ChaSTE will help to measure the vertical temperature gradient and thermal conductivity within the top $10 \mathrm{~cm}$ of regolith on the lunar surface. ChaSTE consists of a thermal probe including sensors and a heater which will be inserted into the lunar regolith down to a depth of $\sim 10$ $\mathrm{cm}$ at the landing site. ChaSTE operates in two different modes, Passive mode operation in which continuous insitu measurements of temperature at different depths are carried out and Active mode operation in which temperature variations in a set period of time and the regolith's thermal conductivity under contact, are estimated. Harness running from the probe will connect the probe to the electronics place inside the lander. An important aspect of the payload is the design of a precise and wide range temperature measurement Front-End (FE) and the selection of a custom-developed Platinum RTD, PT1000 as a sensing element. It is designed and developed by PRL, Ahmedabad and SPL, Thiruvananthapuram (Durga Prasad, 2016).

\section{Instrument for Lunar Seismic Activity (ILSA)}

ILSA is a triple-axis, MEMS-based seismometer that can detect minute ground displacement, velocity, or acceleration caused by lunar quakes. Its primary objective is to characterize the seismicity around the landing site. ILSA is designed to identify acceleration as low as $100 \mathrm{ng} / \sqrt{\mathrm{Hz}}$ with a dynamic range of $\pm 0.5 \mathrm{~g}$ and a bandwidth of $40 \mathrm{~Hz}$. The dynamic range is met by using two sensors, a coarse-range sensor and a fine-range sensor (ISRO, 2019c).

\section{Rover Payloads}

The Chandrayaan-2 Rover consists of two scientific payloads to study the Moon. The instruments mounted on the Rover are as follow:

\section{Alpha Particle X-ray Spectrometer (APXS)}

APXS is designed with an objective to study the elemental composition of lunar rock and soil near the landing site. It achieves this through the X-ray fluorescence spectroscopy technique, where X-ray or alpha particles are used to excite the surface. The working principle of APXS involves measuring the intensity of characteristic X-rays emitted from the sample due to Alpha Particle Induced X-ray Emission (PIXE) and X-ray Florescence (XRF). APXS uses radioactive Curium (244) metal that emits high-energy, alpha particles as well as Xrays enabling both X-ray emission spectroscopy and X-ray fluorescence spectroscopy. Through these techniques, APXS can detect all major rock-forming elements such as Sodium, Magnesium, Aluminum, Silica, Calcium, Titanium, Iron and some trace elements such as Strontium, Yttrium and Zirconium, spanning the energy range of 0.9 to $16 \mathrm{keV}$. The electronics design of the APXS experiment has shown that the developed system provides energy resolution of $\sim 150 \mathrm{eV}$ at $5.9 \mathrm{keV}$ which is comparable to off the shelf Silicon Drift Detector (SDD) based X-ray spectrometers. APXS consists of two packages namely APXS sensor head and APXS backend electronics. APXS sensor head will be mounted on a robotic arm. On command, the robotic arm brings the sensor head close to the lunar surface without touching the surface and after the measurements, the sensor head is taken back to the parking position. The sensor head assembly contains SDD, six alpha sources and front end electronic circuits such as charge sensitive preamplifier (CSPA), shaper and filter circuits associated with the detector. The sensor head contains a circular disc that holds six alpha sources symmetrically around the disc and the detector at the center. It is designed and developed at PRL, Ahmedabad (Shanmugam et al., 2014).

\section{Laser Induced Breakdown Spectroscope (LIBS)}

LIBS is designed with a prime objective to identify and determine the abundance of elements on the lunar surface near the landing site. This is achieved by firing high-powered laser pulses at various locations and analyzing the radiation emitted by the decaying plasma. LIBS performs simultaneous multi-element determination of the matter in any of its diverse forms, namely, solid, liquid or gas using an intense nanosecond pulse duration of the laser beam of lunar regolith from an in-situ distance of $200 \mathrm{~mm}$ from the surface. The plasma emission emanating from the target surface is collected by a chromatic aberration corrected Collection Optics Unit (COU) and spectra are acquired using an aberration corrected concave holographic grating and linear CCD based spectrograph. The spectrograph supports variable time delay in the range of 1 to $5 \mu$ s and an integration time of $8 \mu \mathrm{s}$ to $1 \mathrm{~ms}$. The LIBS instrument is realized with a weight of $1.2 \mathrm{~kg}$ and having power consumption more than $5 \mathrm{~W}$ and a footprint of $180 \times 150 \times 80 \mathrm{~mm}$. It is designed and developed at Laboratory for Electro-Optic Systems (LEOS), Bengaluru (Laxmiprasad et al., 2016). 
Along with the thirteen scientific payloads Chandrayaan-2 Mission also carries a Laser Retroreflector Array (LRA) provided by the National Aeronautics and Space Administration (NASA) for measuring the accurate distance of Moon from Earth.

\section{Conclusion}

The paper provides an insight into Chandrayaan-2, India's second lunar exploration mission. The mission is India's first attempt to soft-land a lander and a rover on the lunar surface. Also, the Chandrayaan-2 is the nation's first indigenously built lunar expedition to explore the unexplored region of the Moon's South Pole. The mission profile for Chandrayaan-2 is explained. Further, the challenges involved in lunar exploration and soft landing missions are explained. The configuration of the launch vehicle GSLV MK-III M1, Chandrayaan-2 Orbiter, a lander (Vikram) and a rover (Pragyan) are discussed in detail. Additionally, details about the scientific payloads mounted on-board the orbiter, lander and a rover to conduct in-situ experiments on the lunar surface are given. Through this mission, India attempts to expand its footprint in space, foster a new age of discovery, increase our understanding of space, stimulate the advancement of technology, promote global alliances and inspire a future generation of explorers and scientists. As NASA is planning to send the first woman and the next man on the south pole of the Moon through its ongoing crewed spaceflight program Artemis. The discoveries made and data collected by the Chandrayaan- 2 mission will play an essential role in the success of such crewed missions. All these unmanned and manned robotic explorers will pave the way for a giant leap forward in space exploration.

\section{Acknowledgement}

The author would like to thank unknown reviewers for their thoughtful suggestions and comments.

\section{Ethics}

The author declares that there is no conflict of interest regarding the publication of this article.

\section{References}

Amitabh, K.S. and T.P. Srinivasan, 2018. Potential landing sites for chandrayaan-2 lander in southern hemisphere of moon. Proceedings of the 49th Lunar and Planetary Science Conference, (PSC' 18), the Woodlands, Houston.

Annadurai, M., 2017. Chandrayaan-2: Lunar orbiter and lander mission. Proceedings of the 10th IAA Symposium on The Future of Space Exploration: Towards the Moon Village and Beyond, (MVB' 17), Torino, Italy.
Bhardwaj, A., Das, T.P., S.V.P. Mohankumar and S.V. Thampi, 2016. CHACE-2 mass spectrometer aboard chandrayaan-2 orbiter to study the lunar neutral exosphere. Proceedings of the 19th National Space Science Symposium (SSS' 16), At Space Physics Laboratory, Thiruvananthapuram, India.

Durga Prasad, K., 2016. Front-end electronics development for ChaSTE payload onboard chandrayaan-2 lander. Physical Research Laboratory - Annual Report 2015-16.

ISRO, 2019a. The challenges of a moon landing. Department of Space, Indian Space Research Organisation (ISRO).

ISRO, 2019b. GSLV Mk III. Department of Space, Indian Space Research Organisation (ISRO).

ISRO, 2019c. Launch Kit at a glance. Department of Space, Indian Space Research Organisation (ISRO).

Laxmiprasad, A.S., A. Goswami, R.V.L.N. Sridhar and K.A. Lohar, 2016. The LIBS instrument for chandrayaan-2 rover: Engineering model development aspects. Proceedings of the 67th International Astronautical Congress, (IAC' 16), At Goudalajara, Mexico.

Manju, G., 2016. RAMBHA Langmuir Probe (LP) Payload onboard chandrayaan-2 lander: Design and development. Proceedings of the 19th National Space Science Symposium, (SSS' 16), Thiruvananthapuram, India.

Narendranath, S., P.S. Athiray, P. Sreekumar, V. Radhakrishnaa and A. Tyagi et al., 2014. Mapping lunar surface chemistry: New prospects with the Chandrayaan-2 Large Area Soft X-ray Spectrometer (CLASS). Adv. Space Res., 54: 1993-1999. DOI: 10.1016/j.asr.2013.04.008

Putrevu, D., A. Das, J.G. Vachhani, S. Trivedi and T. Misra, 2016. Chandrayaan-2 dual-frequency SAR: Further investigation into lunar water and regolith. Adv. Space Res., 57: 627-646. DOI: 10.1016/j.asr.2015.10.029

Rekha, A.R., A. Abdul Shukkoor and P.P. Mohanlal, 2011. Challenges in navigation system design for lunar soft landing. Proceedings of the ISRO Inertial Systems - Thiruvananthapuram Conference, (STC' 11).

Shanmugam, M., S.V.S. Murty, Y.B. Acharya, S.K. Goyal and A.R. Patel et al., 2014. Alpha Particle Xray Spectrometer (APXS) on-board chandrayaan-2 rover. Adv. Space Res., 54: 1974-1984.

DOI: 10.1016/j.asr.2013.03.011

Vadawale, S.V., M. Shanmugam, Y.B. Acharya, A.R. Patel and S.K. Goyal et al., 2014. Solar X-ray Monitor (XSM) on-board Chandrayaan-2 Orbiter. Adv. Space Res., 54: 2021-2028.

DOI: 10.1016/j.asr.2013.06.002 
VSSC, 2019. Results from chandrayaan-1 mission. Vikram Sarabhai Space Centre (VSSC).

\section{Figures Sources}

Fig. https://www.isro.gov.in/sites/default/files/galleries/Chan drayaan-2\%20Mission\%20Gallery/liftoffimage11.jpg

Fig. 2: https://www.isro.gov.in/gslv-mk-iii-m1chandrayaan-2-mission/launch-kit-glance/pages8-9

Fig. 3: M. Annadurai, "Chandrayaan-2: Lunar Orbiter \& Lander Mission", 10th IAA Symposium on The Future of Space Exploration: Towards the Moon Village and Beyond, Torino, Italy, June 2017.

Fig. 4: Author created image (Credit - NASA, https://lunar.gsfc.nasa.gov/)

Fig. 5: https://www.isro.gov.in/gslv-mk-iii-m1chandrayaan-2-mission/launch-kit-glance/pages6-7

Fig. 6: https://www.isro.gov.in/sites/default/files/galleries/Chan drayaan-

2\%20Mission\%20Gallery/01 vikramlandermountedontop oftheorbiterofchandrayaan2.jpg
Fig.

7:

https://www.isro.gov.in/sites/default/files/galleries/Chan drayaan-

2\%20Mission\%20Gallery/04chandrayaan2orbiteratlaunc hcentre.jpg

Fig.

8:

: https://www.isro.gov.in/sites/default/files/galleries/Chan drayaan-

2\%20Mission\%20Gallery/03pragyanrovermountedonthe rampprojectingfromoutofthesidesofvikramlander.jpg

Fig. 9: https://www.isro.gov.in/sites/default/files/galleries/Chan drayaan-

2\%20Mission\%20Gallery/03pragyanrovermountedonthe rampprojectingfromoutofthesidesofvikramlander.jpg

Fig. 10: https://www.isro.gov.in/gslv-mk-iii-m1chandrayaan-2-mission/launch-kit-glance/pages18-21 